\title{
Nonionic surfactant-templated mesoporous carbon as an electrocatalyst support for methanol oxidation
}

\begin{abstract}
Two carbons were synthesized for use as platinum electrocatalyst supports for methanol oxidation. For both materials, furfuryl alcohol was used as the carbon precursor; however, one (CPEG) was made using poly ethylene glycol as the pore former, while the other (CSRF) was produced using Pluronic ${ }^{\circledR}$ F127 as the soft template by organic-organic self-assembly. The CPEG and CSRF carbons were estimated from nitrogen physisorption experiments to be micro- and mesoporous, respectively. Platinum nanoparticles were deposited on each carbon as well as on Vulcan $\mathrm{XC}-72$ carbon by the formic acid reduction method. The physicochemical properties of electrocatalysts were studied using X-ray diffraction (XRD), transmission electron microscopy (TEM), and energy dispersive X-ray analysis (EDX), and their electrochemical features were examined using cyclic voltammetry, chronoamperometry, and impedance spectroscopy. It was found that higher methanol oxidation peak current densities as well as lesser charge transfer resistance at electrode/electrolyte interface were obtained for Pt supported on CSRF as compared to those on Vulcan XC-72 carbon, owing to the higher specific surface area and larger total pore volume $(696 \mathrm{~m} 2 \mathrm{~g}-1$ and $0.60 \mathrm{~cm} 3 \mathrm{~g}-1$, respectively) together with superior electrical conductivity of mesoporous CSRF. On the other hand, the lower surface area and pore volume of microporous CPEG substrate confined $\mathrm{Pt}$ nanoparticles deposition and thus made CPEG-supported $\mathrm{Pt}$ an inefficient methanol oxidation electrocatalyst.
\end{abstract}

Keyword: Pt electrocatalysts; Mesoporous carbon; Nonionic surfactant; Organic-organic self-assembly; Methanol oxidation 